Article

\title{
Evolution of Phenolic Compound Profiles and Antioxidant Activity of Syrah Red and Sparkling Moscatel Wines Stored in Bottles of Different Colors
}

\author{
Joyce Fagundes de Souza ${ }^{1}$, Antonio Mendes de Souza Nascimento ${ }^{1}{ }^{[}$, \\ Maria do Socorro Silva Linhares ${ }^{2}$, Maria da Conceição Prudêncio Dutra ${ }^{1}$, \\ Marcos dos Santos Lima ${ }^{3, *}$ (D) and Giuliano Elias Pereira ${ }^{4}$ \\ 1 Department of Technology and Social Sciences (DTCS III), Campus Juazeiro, Bahia State University, \\ CEP 48905-680 Juazeiro, BA, Brazil; joyce.fagundes08@gmail.com (J.F.d.S.); \\ antonioenologia@gmail.com (A.M.d.S.N.); maria.cp.dutra@gmail.com (M.d.C.P.D.) \\ 2 Graduate Program in Food Science and Technology (PROCTA), Campus Aracaju, Federal University of \\ Sergipe, CEP 49100-000 Sergipe, SE, Brazil; silvinhalinhares2010@hotmail.com \\ 3 Department of Food Technology, Campus Petrolina, Federal Institute of Sertão Pernambucano, Rod. BR 407 \\ Km 08, S/N, Jardim São Paulo, CEP 56314-520 Petrolina, PE, Brazil \\ 4 Brazilian Agricultural Research Corporation (Embrapa), Semiarid/Grapes and Wine, Rodovia Br 428, \\ Km 152, P.O. Box 23, CEP 56302-970 Petrolina, PE, Brazil; giuliano.pereira@embrapa.br \\ * Correspondence: marcos.santos@ifsertao-pe.edu.br; Tel.: +55-87-2101-4330; Fax: +55-87-2101-4300
}

Received: 2 September 2018; Accepted: 9 November 2018; Published: 15 November 2018

\begin{abstract}
The objective of this study was to evaluate the effects of storage time and bottle color on the phenolic compound profiles of Syrah red and sparkling Moscatel wines stored for 12 months in green, amber, and clear bottles. The profile of the phenolic compounds and their antioxidant activity in vitro were determined. Commercial wines were bottled in an automatic filling machine and closed with natural cork. After the bottling process, the wines were stored vertically on shelves which received natural light indirectly $\left( \pm 8 \mathrm{~h}\right.$ /day), at temperatures which varied from 24 to $30{ }^{\circ} \mathrm{C}$ and relative humidity $40-65 \%$. The wines were analyzed every three months over one year. Several phenolic compound families were quantified through reversed-phase high performance liquid chromatography (RP-HPLC) coupled to diode-array detection (DAD) and fluorescence detection (FD). The different bottle colors studied had not influenced the evolution of the sparkling Moscatel and Syrah red wines. The main variations obtained were related to storage time. The main changes were observed in the Syrah wine, where storage time was associated with an increase in hue $\left(\mathrm{h}^{*}\right)$, decrease in catechin and epicatechin, and most notably, a decrease in the anthocyanin malvidin 3-glucoside. The sparkling Moscatel wine did not show important changes in most phenolic compounds; however, the catechin increased significantly during storage and this increase was similar in bottles of all colors. In general, the wines were stable in relation to the antioxidant activity in vitro.
\end{abstract}

Keywords: bottle color; shelf life; Vitis vinifera L.; quality control

\section{Introduction}

The wine sector has increasingly sought to obtain wines with high sensory quality as well as typical characteristics, since these attributes are the main aspects expected by consumers [1]. Products which do not fulfill the sensory expectations of the consumers may be rejected.

The São Francisco Valley (SFV), located in the Northeast Region of Brazil, is an unusual and emerging area [2] for wine grape cultivation. This region currently produces 2 million liters of sparkling Moscatel wines and 1.16 million liters of young red wines (mainly with Syrah grapes). 
The characteristics of these wines make them appropriate for rapid consumption, and storage is not recommended to extend for more than two years [3].

The shelf life of a wine can be difficult to assess, since its chemical composition is very complex [4] and a number of favorable or unfavorable reactions can occur during storage time. In addition, temperature, bottle position, exposure to light, moisture and container color may affect the qualitative characteristics of the wine. Furthermore, these factors may promote changes in the phenolic composition through copigmentation, polymerization and oxidation reactions. These reactions may have a negative or positive impact from the consumer's viewpoint $[5,6]$.

Some authors have evaluated the factors related to wine aging, such as: the adverse influence of light and the influence of the bottle color on the evolution of the color and volatile composition of a wine [7-12]. However, studies to measure the effect of bottle colors on the profile of several families of phenolic compounds related to wine quality, such as flavanols, flavonols, phenolic acids, anthocyanins, and stilbenes, have not been previously reported.

Another parameter directly influenced by storage is the antioxidant activity in vitro, and some studies have shown that there is a considerable decrease over time $[4,13]$.

Given this, the objective of the present study was to evaluate the evolution of Syrah red and sparkling Moscatel wines stored for 12 months in bottles of different colors, in relation to the phenolic compound profile obtained through RP-HPLC/DAD/FD and the antioxidant activity in vitro.

\section{Materials and Methods}

\subsection{Chemicals}

Trolox (6-hydroxy-2,5,7,8-tetramethylchroman-2-carboxylic acid), 2,2-diphenyl-1-picrylhydrazyl (DPPH) and 2,2'-azino-bis (3-ethylbenzothiazoline-6-sulfonic acid) (ABTS) were obtained from Sigma-Aldrich (St. Louis, MO, USA). Potassium persulfate, ethanol, acetone, sodium carbonate and Folin-Ciocalteu reagent were obtained from Merck (Darmstadt, Germany). Methanol and acetonitrile (both HPLC grade) and orthophosphoric acid were provided by Vetec Química Fina Ltd.a (Rio de Janeiro, Brazil), JT Baker (Phillipsburg, NJ, USA) and Fluka (Buchs, Switzerland), respectively. The water used in this study was purified with the aid of an Elga PURELAB ${ }^{\circledR}$ Option-Q (ELGA Labwater, Waltham, MA, USA) purification system. The standards of ferulic, cinnamic and gallic acids were obtained from Chem Service (West Chester, PA, USA), and the $p$-coumaric and chlorogenic acids, viniferin and piceatannol were obtained from Sigma-Aldrich (St. Louis, MO, USA). The standards kaempferol 3-glucoside, rutin, pelargonidin 3-glucoside, (+)-catechin, cyanidin 3-glucoside, (-)-epicatechin, (-)-epigallocatechin, (-)-epicatechin gallate, isorhamnetin 3-glucoside, delphinidin 3-glucoside, malvidin 3-glucoside, petunidin 3-glucoside, peonidin 3-glucoside, procyanidin A2, procyanidin B1, procyanidin B2, quercetin 3-glucoside, and cis and trans resveratrol were obtained from Extrasynthese (Genay, France).

\subsection{Samples}

Two types of bottles were acquired from the company Owens-Illinois (Recife, PE, Brazil), one bottle manufactured specifically for sparkling wine (Trilogy Glass \& Packaging Inc., Sparkling wine bottle $750 \mathrm{~mL}$ Prosecco) and the other for table wine (BD Reference, $750 \mathrm{~mL}$ Bordeaux), both with a holding capacity of $750 \mathrm{~mL}$. These were obtained in clear, green and amber-colored glass. Single varietal Syrah (red) and sparkling Moscatel (white) wines from the same commercial production tank were obtained from the Miolo Wine Group (Casa Nova, BA, Brazil) located in the São Francisco Valley (SFV; latitude $9^{\circ} 15^{\prime} \mathrm{S}$; longitude $40^{\circ} 50^{\prime} \mathrm{W}$ ). The bottling was carried out by the cited company with the aid of an automatic filling machine, Suprema 30 (Bertolasso, Italy). After the bottling process, the bottles were corked (natural cork) and stored vertically on shelves which received natural light indirectly ( $\pm 8 \mathrm{~h} /$ day), at temperatures which varied from 24 to $30^{\circ} \mathrm{C}$ and relative humidity $40-65 \%$, for a period of 12 months. All bottles were placed standing at the front of shelves so as to receive 
the ambient light in an identical manner. The treatments for the Syrah red wine were as follows: stored in clear bottles and evaluated after 3, 6, 9, and 12 months (SCB3, SCB6, SCB9, and SCB12, respectively); stored in green bottles and evaluated after 3, 6, 9 and 12 months (SGB3, SGB6, SGB9 and SBG12, respectively); and stored in amber bottles and evaluated after 3, 6, 9, and 12 months of storage (SAB3, SAB6, SAB9, and SAB12, respectively). The treatments for the sparkling Moscatel wine were as follows: stored in clear bottles and evaluated after 3, 6, 9, and 12 months (MCB3, MCB6, MCB9, and MCB12, respectively); stored in green bottles and evaluated after 3, 6, 9, and 12 months (MGB3, MGB6, MGB9, and MGB12, respectively); and stored in amber bottles and evaluated after 3, 6, 9, and 12 months (MAB3, MAB6, MAB9, and MAB12, respectively). For each storage time (reference $0,3,6,9$, and 12 months), three bottles were evaluated corresponding to three replicates, totaling 45 bottles for Syrah and 45 bottles for sparkling Moscatel with respect to the three bottle colors.

\subsection{Basic Parameters, CIE L*a* $b^{*}$ Color, Total Phenolic and Monomeric Anthocyanins}

The determination of the density, alcohol content, $\mathrm{pH}$, titratable acidity and free and total sulfur dioxide was carried out following the methods recommended by the International Organization of Vine and Wine [14]. The color was measured with the aid of the coordinates $L^{*}, a^{*}, b^{*}, C^{*}$ and $h^{*}$ (CIELab) in a colorimeter, model Delta Vista $450 \mathrm{G}$, with the software program i7 (Delta Color, Brazil), using an acrylic cuvette with a $1 \mathrm{~cm}$ optical path length.

The total phenolic content was determined using the colorimetric method of Folin-Ciocalteu [15], with gallic acid as the standard The results were expressed as the gallic acid equivalent (GAE $\mathrm{mg} \mathrm{L}^{-1}$ ).

For the determination of the monomeric anthocyanins, the differential $\mathrm{pH}$ method was used [16], and the values were expressed as malvidin 3-glucoside in $\mathrm{mg} \mathrm{L}^{-1}$.

\subsection{Antioxidant Activity In Vitro}

The antioxidant activity (AOX) of the wines was measured through the methods of free radical capture using 2,2-diphenyl-1-picrylhydrazyl (DPPH) and 2,2'-azino-bis (3-ethylbenzothiazoline6-sulfonic acid) (ABTS). In both methods, the analytical standard Trolox was used to construct the calibration curves, following the methods described by Kim, Guo, Packer [17], and Re et al. [18]. The results were expressed as equivalent in millimoles of Trolox per liter of wine (mmol TE L $\left.{ }^{-1}\right)$.

\subsection{Determination of Phenolic Compounds Profile by RP-HPLC/DAD/FD}

The individual phenolic compounds in the wines were determined by reversed-phase high performance liquid chromatography (RP-HPLC) on a Waters Systems (model Alliance e2695, Milford, MA, USA) coupled to diode array detection (DAD) and fluorescence detection (FD). The analysis was performed according to the methodology described by Silva et al. [19], using the software program Empower $^{\mathrm{TM}} 2$ (Milford, MA, USA) for data treatment. For the separation of compounds, a Gemini NX C-18 column $(150 \mathrm{~mm} \times 4.6 \mathrm{~mm} \times 3 \mu \mathrm{m})$ and a Gemini NX C-18 guard column $(4.0 \mathrm{~mm} \times 3 \mathrm{~mm} \times$ $3 \mu \mathrm{m}$ ) were used, both manufactured by Phenomenex (Torrance, CA, USA).

The oven temperature was maintained at $40{ }^{\circ} \mathrm{C}$ and the solvent flow at $0.6 \mathrm{~mL} \mathrm{~min}^{-1}$, with a total run time of $65 \mathrm{~min}$. The gradient used was $0 \mathrm{~min}: 100 \% \mathrm{~A} ; 18 \mathrm{~min}: 87.5 \% \mathrm{~A}, 2.5 \% \mathrm{~B}, 10.0 \% \mathrm{C}$; $30 \mathrm{~min}: 83.5 \% \mathrm{~A}, 3.2 \% \mathrm{~B}, 13.3 \% \mathrm{C}$; $36 \mathrm{~min}: 75.0 \% \mathrm{~A}, 5.0 \% \mathrm{~B}, 20.0 \% \mathrm{C} ; 48.5 \mathrm{~min}: 65.0 \% \mathrm{~A}, 8.3 \% \mathrm{~B}$, $26.7 \%$ C; 50 min: $65.0 \%$ A, 8.3\% B, 26.7\% C; and $65 \mathrm{~min}: 100 \%$ A. Solvent A consisted of a solution of $25 \mathrm{mmol} \mathrm{L}^{-1}$ of potassium dihydrogenphosphate with the $\mathrm{pH}$ adjusted to 2.05 with phosphoric acid, solvent $B$ was methanol and solvent $C$ was acetonitrile. In the DAD, the detection of the compounds was performed at: $280 \mathrm{~nm}$ for gallic acid, (-)-epicatechin gallate, (-)-epigallocatechin, and cis-resveratrol; $320 \mathrm{~nm}$ for p-coumaric acid, ferulic acid, caftaric acid, chlorogenic acid, caffeic acid, piceatannol, viniferin, and trans-resveratrol; $360 \mathrm{~nm}$ for the flavonols: kaempferol, rutin, isorhamnetin, myricetin, and quercetin; and $520 \mathrm{~nm}$ for the anthocyanins: delphinidin 3-O-glucoside, cyanidin 3-O-glucoside, pelargonidin 3-O-glucoside, peonidin 3-O-glucoside, petunidin 3-glucoside, and malvidin 3-O-glucoside. In the FD, the photon excitation was carried out at $280 \mathrm{~nm}$ and the 
emission at $320 \mathrm{~nm}$ for procyanidin B1, B2 and A2, epicatechin, and catechin. The detection and quantification of the compounds was carried out through the use of external standards.

\subsection{Statistical Analysis}

For each storage time, three bottles were evaluated, and all analyses carried out in triplicate. The results obtained were submitted to analysis of variance (one-way ANOVA) and compared applying the Tukey test $(p<0.05)$, with the aid of the software program SPSS Inc., version 17.0 (Chicago, IL, USA). The results were expressed as average \pm standard deviation. In order to evaluate the behavior of the phenolic profile in relation to the storage time in the different colored bottles, the application of multivariate statistics was carried out using principal component analysis (PCA), as described by Hongyu, Sandanielo and Junior [20].

\section{Results and Discussion}

\subsection{Basic Parameters of Wines}

The Syrah red and sparkling Moscatel wines were analyzed before their bottling to determine the basic characteristics of the samples (Table 1) using classic analyses described by the International Organization of Vine and Wine (OIV) [21].

Table 1. Basic parameters (mean \pm standard deviation) of the sparkling Moscatel and Syrah wines.

\begin{tabular}{ccc}
\hline Basic Parameters & Sparkling Moscatel & Syrah \\
\hline $\mathrm{pH}$ & $3.40 \pm 0.02$ & $3.90 \pm 0.02$ \\
Titratable acidity $\left(\mathrm{g} \mathrm{L}^{-1}\right)$ & $7.6 \pm 0.1$ & $5.2 \pm 0.2$ \\
Alcohol content $(\% / v)$ & $9.1 \pm 0.1$ & $13.1 \pm 0.1$ \\
Volatile acidity $\left(\mathrm{g} \mathrm{L}^{-1}\right)$ & $0.5 \pm 0.1$ & $0.6 \pm 0.1$ \\
Free $\mathrm{SO}_{2}\left(\mathrm{mg} \mathrm{L}^{-1}\right)$ & $47 \pm 2$ & $52 \pm 2$ \\
Total $\mathrm{SO}_{2}\left(\mathrm{mg} \mathrm{L}^{-1}\right)$ & $159 \pm 1$ & $96 \pm 1$ \\
\hline
\end{tabular}

\subsection{Color Evolution Based on the CIE $L^{*} a^{*} b^{*}$ System}

The results for the wine color are given in Table 2. For the sparkling Moscatel, there was a similar increase in the $b^{*}$ and $C^{*}$ values for the three bottle colors, with ranges of $6.9-12.87$ and 7.0-12.87 for $b^{*}$ and $C^{*}$, respectively. The increase in the $+b^{*}$ values indicates an increase in the yellow color, which was also observed by Del Caro et al. [8] who studied the stability of Moscato and Malvasia white wines over a period of 18 months. This observation could be due to the oxidation of the flavanols in the wine. The increase in $C^{*}$ values represents an increase in the chroma (higher clarity) over time, which could be due to sedimentation of the colloids in the wine during storage.

In relation to the color of the Syrah wine, a similar decrease in the $\mathrm{a}^{*}$ values during storage was observed for all bottle colors, with values in the range of 7.99-3.48. This decrease in the $\mathrm{a}^{*}$ values reflects a decrease in the red color of the wine and this change is more evident considering the increase in the hue angle $\left(\mathrm{h}^{*}\right.$ ) (in the range of $40.07^{\circ}$ and $64.36^{\circ}$ ). According to Sant'Anna, Gurak, Marczak, and Tessaro [22], an increase in the $h^{*}$ values indicates an increase in the orange hue, which is evidence of the evolution of a wine. Marquez et al. [10] evaluated the stability of sweet red wines over 12 months and obtained results similar to those of this study. This color change can be attributed to a decrease in the anthocyanin content.

\subsection{Evolution of the Individual Phenolic Compounds}

The profiles for the individual phenolic compounds in the sparkling Moscatel and Syrah red wines are provided in Tables 3 and 4, respectively. 
Table 2. Evolution of CIE L*a*b* color (mean \pm standard deviation) of the sparkling Moscatel and Syrah wines stored in bottles of different colors for 12 months.

\begin{tabular}{|c|c|c|c|c|c|c|c|c|c|c|c|c|c|}
\hline \multirow{2}{*}{ Color } & \multirow{2}{*}{$\begin{array}{c}\text { Reference } \\
\text { (0 Months) }\end{array}$} & \multicolumn{4}{|c|}{ Clear Bottle } & \multicolumn{4}{|c|}{ Green Bottle } & \multicolumn{4}{|c|}{ Amber Bottle } \\
\hline & & 3 Months & 6 Months & 9 Months & 12 Months & 3 Months & 6 Months & 9 Months & 12 Months & 3 Months & 6 Months & 9 Months & 12 Months \\
\hline \multicolumn{14}{|c|}{$\begin{array}{l}\text { Sparkling } \\
\text { Moscatel }\end{array}$} \\
\hline $\mathrm{L}^{*}$ & $54.03 \pm 0.40$ & $53.69 \pm 0.55^{a}$ & $53.75 \pm 0.34^{\mathrm{a}}$ & $53.89 \pm 0.42^{\mathrm{a}}$ & $54.21 \pm 0.06^{\mathrm{a}}$ & $53.36 \pm 0.08 \mathrm{ab}$ & $53.58 \pm 0.31^{\mathrm{a}}$ & $53.63 \pm 0.48^{a}$ & $54.08 \pm 0.26^{\mathrm{a}}$ & $54.08 \pm 0.12^{\mathrm{a}}$ & $52.47 \pm 0.09^{b}$ & $53.53 \pm 0.15^{a}$ & $53.53 \pm 0.45^{\mathrm{a}}$ \\
\hline$a^{*}$ & $-1.12 \pm 0.21$ & $-1.10 \pm 0.20^{\mathrm{a}}$ & $-1.45 \pm 0.23^{\mathrm{a}}$ & $-1.65 \pm 0.16 \mathrm{ab}$ & $-2.04 \pm 0.04 \mathrm{bc}$ & $-1.58 \pm 0.06 \mathrm{ab}$ & $-1.64 \pm 0.17 \mathrm{ab}$ & $-1.67 \pm 0.15 \mathrm{ab}$ & $-2.15 \pm 0.03^{c}$ & $-1.62 \pm 0.10 \mathrm{ab}$ & $1.51 \pm 0.25^{\mathrm{a}}$ & $-1.72 \pm 0.18 \mathrm{abc}$ & $-2.05 \pm 0.03 \mathrm{bc}$ \\
\hline$b^{*}$ & $6.90 \pm 0.47$ & $8.05 \pm 0.50 \mathrm{de}$ & $8.94 \pm 1.11$ bcde & $9.44 \pm 0.73 \mathrm{bcd}$ & $11.87 \pm 0.23 \mathrm{a}$ & $8.35 \pm 0.10^{\text {cde }}$ & $9.50 \pm 0.26 \mathrm{bc}$ & $10.20 \pm 0.16^{b}$ & $12.70 \pm 0.31^{\mathrm{a}}$ & $7.74 \pm 0.24 \mathrm{e}$ & $9.71 \pm 0.58 \mathrm{bc}$ & $10.20 \pm 0.24 \mathrm{~b}$ & $12.45 \pm 0.19^{a}$ \\
\hline $\mathrm{C}^{*}$ & $7.00 \pm 0.44$ & $8.20 \pm 0.54^{\mathrm{d}}$ & $9.39 \pm 0.68 \mathrm{bc}$ & $9.59 \pm 0.74 \mathrm{bc}$ & $12.04 \pm 0.23^{a}$ & $8.49 \pm 0.11 \mathrm{~cd}$ & $9.64 \pm 0.29 \mathrm{~b}$ & $10.33 \pm 0.14^{b}$ & $12.88 \pm 0.31^{\mathrm{a}}$ & $7.91 \pm 0.22 \mathrm{~d}$ & $10.16 \pm 0.03^{b}$ & $10.35 \pm 0.23^{b}$ & $12.62 \pm 0.19^{a}$ \\
\hline \multicolumn{14}{|l|}{$\begin{array}{c}\text { Syrah red } \\
\text { wine }\end{array}$} \\
\hline $\mathrm{L}^{*}$ & $17.55 \pm 1.11$ & $13.48 \pm 0.34 \mathrm{~cd}$ & $14.24 \pm 0.37 \mathrm{bc}$ & $15.82 \pm 0.27^{\mathrm{a}}$ & $13.97 \pm 0.22 \mathrm{bcd}$ & $13.62 \pm 0.49 \mathrm{bcd}$ & $14.10 \pm 0.03 \mathrm{bc}$ & $13.57 \pm 0.1 \mathrm{bcd}$ & $13.66 \pm 0.09 \mathrm{bcd}$ & $14.36 \pm 0.31^{\mathrm{b}}$ & $13.24 \pm 0.09 \mathrm{~d}$ & $15.91 \pm 0.26^{\mathrm{a}}$ & $13.75 \pm 0.38 \mathrm{bcc}$ \\
\hline $\begin{array}{l}L \\
a^{*}\end{array}$ & $7.99 \pm 0.57$ & $4.47 \pm 0.38 \mathrm{bcd}$ & $4.34 \pm 0.37 \mathrm{cde}$ & $3.52 \pm 0.05 \mathrm{de}$ & $3.48 \pm 0.44^{\mathrm{e}}$ & $4.33 \pm 0.23 \mathrm{cde}$ & $4.39 \pm 0.21$ cde & $5.49 \pm 0.46^{\mathrm{a}}$ & $4.49 \pm 0.35^{\mathrm{e}}$ & $5.39 \pm 0.33 \mathrm{ab}$ & $4.59 \pm 0.21 \mathrm{abc}$ & $4.56 \pm 0.39 \mathrm{abc}$ & $3.69 \pm 0.26^{\text {cde }}$ \\
\hline$b^{*}$ & $6.74 \pm 0.84$ & $6.51 \pm 0.27^{\mathrm{a}}$ & $6.60 \pm 0.28^{a}$ & $5.02 \pm 0.04^{c}$ & $6.08 \pm 0.43 \mathrm{ab}$ & $6.27 \pm 0.24 \mathrm{ab}$ & $6.56 \pm 0.09^{a}$ & $6.59 \pm 0.19^{\mathrm{a}}$ & $6.29 \pm 0.24^{a}$ & $6.27 \pm 0.40 \mathrm{ab}$ & $6.67 \pm 0.13^{a}$ & $5.49 \pm 0.38 \mathrm{bc}$ & $6.37 \pm 0.21^{a}$ \\
\hline $\mathrm{C}^{*}$ & $10.46 \pm 0.90$ & $7.39 \pm 0.27 \mathrm{~cd}$ & $8.46 \pm 0.21 \mathrm{ab}$ & $6.39 \pm 0.20^{d}$ & $6.39 \pm 0.35 \mathrm{~d}$ & $8.45 \pm 0.37 \mathrm{ab}$ & $8.44 \pm 0.44 \mathrm{ab}$ & $7.78 \pm 0.21 \mathrm{bc}$ & $7.54 \pm 0.34 \mathrm{bc}$ & $7.69 \pm 0.41 \mathrm{bc}$ & $8.45 \pm 0.18 \mathrm{ab}$ & $8.84 \pm 0.56^{\mathrm{a}}$ & $7.36 \pm 0.31^{\mathrm{cd}}$ \\
\hline $\mathrm{h}^{*}$ & $40.07 \pm 2.54$ & $57.74 \pm 0.26^{b c}$ & $53.99 \pm 0.90$ cde & $54.72 \pm 0.46$ cde & $64.36 \pm 4.31^{\mathrm{a}}$ & $55.14 \pm 0.68$ bcde & $52.40 \pm 1.26$ de & $58.01 \pm 0.27 \mathrm{bc}$ & $56.52 \pm 0.82 \mathrm{bcd}$ & $51.26 \pm 0.15^{\mathrm{e}}$ & $54.47 \pm 2.67$ cde & $53.15 \pm 2.17 \mathrm{cde}$ & $59.92 \pm 1.16 \mathrm{ab}$ \\
\hline
\end{tabular}

Averages followed by the same letters on a line do not differ between themselves according to the Tukey test $(p<0.05)$. 
Table 3. Evolution of phenolic compounds $\left(\mathrm{mg} \mathrm{L}^{-1}\right)$ (mean \pm standard deviation) in the sparkling Moscatel stored in bottles of different colors for 12 months.

\begin{tabular}{|c|c|c|c|c|c|c|c|c|c|c|c|c|c|}
\hline \multirow{2}{*}{ Compounds } & \multirow{2}{*}{$\begin{array}{l}\text { Reference } \\
\text { (0 Months) }\end{array}$} & \multicolumn{4}{|c|}{ Clear Bottle } & \multicolumn{4}{|c|}{ Green Bottle } & \multicolumn{4}{|c|}{ Amber Bottle } \\
\hline & & 3 Months & 6 Months & 9 Months & 12 Months & 3 Months & 6 Months & 9 Months & 12 Months & 3 Months & 6 months & 9 Months & 12 Months \\
\hline \multicolumn{14}{|l|}{ Flavonols } \\
\hline Kaempferol & $0.2 \pm 0.0$ & $0.1 \pm 0.0^{b}$ & $0.2 \pm 0.0^{\mathrm{a}}$ & $0.2 \pm 0.0^{\mathrm{a}}$ & $0.2 \pm 0.0^{\mathrm{a}}$ & $0.1 \pm 0.0^{b}$ & $0.2 \pm 0.0^{\mathrm{a}}$ & $0.2 \pm 0.0^{\mathrm{a}}$ & $0.2 \pm 0.0^{\mathrm{a}}$ & $0.2 \pm 0.0^{\mathrm{a}}$ & $0.2 \pm 0.0^{\mathrm{a}}$ & $0.2 \pm 0.0^{\mathrm{a}}$ & $0.2 \pm 0.0^{\mathrm{a}}$ \\
\hline Quercetin & $1.4 \pm 0.2$ & $0.4 \pm 0.0^{\mathrm{d}}$ & $1.0 \pm 0.2 \mathrm{abc}$ & $0.9 \pm 0.1^{\mathrm{c}}$ & $1.1 \pm 0.0^{\mathrm{ab}}$ & $0.3 \pm 0.0^{\mathrm{d}}$ & $1.0 \pm 0.7^{b c}$ & $0.9 \pm 0.1^{c}$ & $1.2 \pm 0.1^{\mathrm{a}}$ & $1.0 \pm 0.0^{b c}$ & $0.9 \pm 0.1 \mathrm{bc}$ & $0.9 \pm 0.1^{\mathrm{c}}$ & $1.1 \pm 0.1 \mathrm{ab}$ \\
\hline Isorhamnetin & $0.1 \pm 0.0$ & $0.12 \pm 0.0^{\mathrm{b}}$ & $0.1 \pm 0.0^{\mathrm{a}}$ & $0.1 \pm 0.0^{\mathrm{ab}}$ & $0.1 \pm 0.0^{\mathrm{a}}$ & $0.1 \pm 0.0^{\mathrm{a}}$ & $0.1 \pm 0.0^{\mathrm{a}}$ & $0.1 \pm 0.0^{\mathrm{a}}$ & $0.1 \pm 0.0^{\mathrm{ab}}$ & $0.1 \pm 0.0^{\mathrm{a}}$ & $0.1 \pm 0.0^{\mathrm{a}}$ & $0.1 \pm 0.0^{\mathrm{ab}}$ & $0.1 \pm 0.0^{\mathrm{a}}$ \\
\hline Myricetin & $0.2 \pm 0.0$ & $0.2 \pm 0.0^{\mathrm{b}}$ & $0.2 \pm 0.0^{\mathrm{a}}$ & $0.2 \pm 0.0^{\mathrm{a}}$ & $0.2 \pm 0.0^{\mathrm{a}}$ & $0.2 \pm 0.0^{b}$ & $0.2 \pm 0.0^{\mathrm{a}}$ & $0.2 \pm 0.0^{\mathrm{a}}$ & $0.2 \pm 0.0^{\mathrm{a}}$ & $0.2 \pm 0.0^{\mathrm{a}}$ & $0.2 \pm 0.0^{\mathrm{a}}$ & $0.2 \pm 0.0^{\mathrm{a}}$ & $0.2 \pm 0.0^{\mathrm{a}}$ \\
\hline Rutin & $0.2 \pm 0.0$ & $0.1 \pm 0.0^{\mathrm{e}}$ & $0.2 \pm 0.0^{\mathrm{bcd}}$ & $0.2 \pm 0.0^{\mathrm{cd}}$ & $0.2 \pm 0.0^{\mathrm{cd}}$ & $0.1 \pm 0.0^{\mathrm{e}}$ & $0.2 \pm 0.0^{b c}$ & $0.2 \pm 0.0^{\mathrm{ab}}$ & $0.2 \pm 0.0^{\mathrm{e}}$ & $0.2 \pm 0.0^{b c}$ & $0.2 \pm 0.0^{\mathrm{cd}}$ & $0.2 \pm 0.0^{\mathrm{cd}}$ & $0.3 \pm 0.0^{\mathrm{a}}$ \\
\hline$\sum$ Flavonols & 2.1 & 0.9 & 1.7 & 1.6 & 1.8 & 0.9 & 1.7 & 1.7 & 1.8 & 1.7 & 1.7 & 1.6 & 1.9 \\
\hline $\begin{array}{l}\text { Flavanols } \\
\text { Catechin }\end{array}$ & $1.5 \pm 0.5$ & $0.5 \pm 0.0^{c}$ & $1.8 \pm 0.1^{\mathrm{b}}$ & $1.7 \pm 0.1^{\mathrm{b}}$ & $6.0 \pm 0.0^{\mathrm{a}}$ & $0.5 \pm 0.0^{c}$ & $1.8 \pm 0.0^{\mathrm{b}}$ & $1.7 \pm 0.1^{\mathrm{b}}$ & $5.8 \pm 0.0^{\mathrm{a}}$ & $1.7 \pm 0.1^{\mathrm{b}}$ & $1.7 \pm 0.1^{\mathrm{b}}$ & $1.6 \pm 0.2^{b}$ & $5.8 \pm 0.0^{\mathrm{a}}$ \\
\hline Epicatechin & $0.9 \pm 0.2$ & $0.3 \pm 0.0^{\mathrm{e}}$ & $1.0 \pm 0.0^{\mathrm{abc}}$ & $0.9 \pm 0.1 \mathrm{bc}$ & $0.5 \pm 0.1^{\mathrm{d}}$ & $0.3 \pm 0.0^{\mathrm{e}}$ & $1.0 \pm 0.0^{\mathrm{a}}$ & $0.8 \pm 0.0^{b c}$ & $0.9 \pm 0.0 \mathrm{abc}$ & $0.9 \pm 0.0 \mathrm{abc}$ & $0.9 \pm 0.1 \mathrm{abc}$ & $0.8 \pm 0.0^{\mathrm{c}}$ & $0.8 \pm 0.1^{\mathrm{c}}$ \\
\hline Epigallocatechin & $0.7 \pm 0.1$ & $0.4 \pm 0.0^{\mathrm{fg}}$ & $0.7 \pm 0.0^{c}$ & $1.1 \pm 0.0^{\mathrm{b}}$ & $1.2 \pm 0.0^{\mathrm{b}}$ & $0.3 \pm 0.0^{g}$ & $0.4 \pm 0.0^{\text {efg }}$ & $0.5 \pm 0.0^{\text {cdef }}$ & $1.4 \pm 0.1^{\mathrm{a}}$ & $0.6 \pm 0.0^{\mathrm{cd}}$ & $0.7 \pm 0.2^{c}$ & $0.5 \pm 0.0^{\text {cde }}$ & $1.2 \pm 0.0^{\mathrm{b}}$ \\
\hline Epicatechin gallate & $0.7 \pm 0.0$ & $0.5 \pm 0.0^{\mathrm{fg}}$ & $0.8 \pm 0.0^{\mathrm{cd}}$ & $0.9 \pm 0.0^{\mathrm{a}}$ & $0.5 \pm 0.1 \mathrm{fg}$ & $0.5 \pm 0.0^{\mathrm{fg}}$ & $0.6 \pm 0.0^{\mathrm{e}}$ & $0.8 \pm 0.0^{b}$ & $0.5 \pm 0.0 \mathrm{~g}$ & $0.7 \pm 0.0^{\mathrm{d}}$ & $0.7 \pm 0.0^{\mathrm{d}}$ & $0.8 \pm 0.0 \mathrm{bc}$ & $0.6 \pm 0.0^{\mathrm{f}}$ \\
\hline \multirow{2}{*}{\multicolumn{14}{|c|}{ Proanthocyanidins }} \\
\hline & & & & & & & & & & & & & \\
\hline Procyanidin A2 & $0.4 \pm 0.0$ & $0.3 \pm 0.0^{\mathrm{fg}}$ & $0.4 \pm 0.0 \mathrm{de}$ & $0.5 \pm 0.0^{\mathrm{a}}$ & $0.3 \pm 0.0^{\mathrm{f}}$ & $0.2 \pm 0.0 \mathrm{~g}$ & $0.4 \pm 0.0^{\mathrm{bcd}}$ & $0.4 \pm 0.0^{\mathrm{bc}}$ & $0.3 \pm 0.0^{\mathrm{f}}$ & $0.4 \pm 0.0^{\mathrm{e}}$ & $0.4 \pm 0.0^{\text {cde }}$ & $0.5 \pm 0.0 \mathrm{ab}$ & $0.3 \pm 0.0^{\mathrm{f}}$ \\
\hline Procyanidin B1 & $1.0 \pm 0.3$ & $0.3 \pm 0.0^{\mathrm{de}}$ & $0.7 \pm 0.0^{b c}$ & $0.5 \pm 0.1$ de & $3.7 \pm 0.1^{\mathrm{a}}$ & $0.3 \pm 0.0^{\mathrm{e}}$ & $0.6 \pm 0.0^{c}$ & $0.5 \pm 0.0^{\mathrm{d}}$ & $3.6 \pm 0.1^{a}$ & $0.8 \pm 0.0^{b}$ & $0.6 \pm 0.0^{c}$ & $0.4 \pm 0.1 \mathrm{de}$ & $3.7 \pm 0.1^{\mathrm{a}}$ \\
\hline Procyanidin B2 & $4.1 \pm 0.6$ & $1.4 \pm 0.0^{c}$ & $6.1 \pm 0.1^{\mathrm{a}}$ & $6.0 \pm 0.3^{\mathrm{a}}$ & $2.4 \pm 0.0^{\mathrm{b}}$ & $1.4 \pm 0.0^{c}$ & $6.1 \pm 0.2^{a}$ & $5.8 \pm 0.3^{\mathrm{a}}$ & $2.3 \pm 0.1^{\mathrm{b}}$ & $5.6 \pm 0.2^{a}$ & $5.8 \pm 0.3^{\mathrm{a}}$ & $6.0 \pm 0.1^{\mathrm{a}}$ & $2.4 \pm 0.1^{b}$ \\
\hline \multirow{2}{*}{\multicolumn{14}{|c|}{$\begin{array}{l}2 \text { Proanthocyanidins } \\
\text { Phenolic acids }\end{array}$}} \\
\hline & & & & & & & & & & & & & \\
\hline Gallic acid & $1.1 \pm 0.4$ & $0.4 \pm 0.0 \mathrm{~g}$ & $1.8 \pm 0.0^{\mathrm{abc}}$ & $1.8 \pm 0.0^{\mathrm{ab}}$ & $1.5 \pm 0.0^{\text {cde }}$ & $0.4 \pm 0.0 \mathrm{~g}$ & $1.7 \pm 0.0^{\mathrm{bcd}}$ & $1.9 \pm 0.0^{\mathrm{a}}$ & $1.4 \pm 0.0^{\mathrm{f}}$ & $1.6 \pm 0.0^{\mathrm{def}}$ & $1.8 \pm 0.1 \mathrm{abc}$ & $1.5 \pm 0.0^{\text {ef }}$ & $1.4 \pm 0.1^{\mathrm{ef}}$ \\
\hline Caffeic acid & $1.6 \pm 0.0$ & $0.5 \pm 0.0^{\mathrm{d}}$ & $1.8 \pm 0.1 \mathrm{abc}$ & $1.9 \pm 0.0 \mathrm{ab}$ & $2.0 \pm 0.0^{\mathrm{ab}}$ & $0.5 \pm 0.0^{\mathrm{d}}$ & $1.9 \pm 0.0^{\mathrm{ab}}$ & $2.0 \pm 0.1 \mathrm{ab}$ & $2.1 \pm 0.1^{\mathrm{a}}$ & $1.6 \pm 0.0^{c}$ & $1.8 \pm 0.1 \mathrm{bc}$ & $1.9 \pm 0.2^{\mathrm{ab}}$ & $2.1 \pm 0.1^{a}$ \\
\hline Caftaric acid & $57.5 \pm 1.7$ & $18.1 \pm 0.5^{\mathrm{d}}$ & $71.8 \pm 0.6^{\mathrm{ab}}$ & $66.8 \pm 1.6 \mathrm{abc}$ & $64.8 \pm 1.5^{\mathrm{c}}$ & $17.6 \pm 0.1^{d}$ & $73.2 \pm 1.5^{\mathrm{a}}$ & $66.4 \pm 1.4 \mathrm{abc}$ & $66.3 \pm 1.3^{\mathrm{bc}}$ & $71.8 \pm 2.5 \mathrm{ab}$ & $71.7 \pm 3.7^{\mathrm{ab}}$ & $65.3 \pm 5.7^{\mathrm{bc}}$ & $67.4 \pm 0.7 \mathrm{abc}$ \\
\hline Chlorogenic acid & $1.4 \pm 0.2$ & $0.4 \pm 0.0^{\mathrm{e}}$ & $1.1 \pm 0.1^{\mathrm{cd}}$ & $0.8 \pm 0.0^{\mathrm{d}}$ & $3.9 \pm 0.1^{\mathrm{a}}$ & $0.4 \pm 0.0^{\mathrm{e}}$ & $1.1 \pm 0.0^{b c}$ & $0.9 \pm 0.1^{\mathrm{d}}$ & $3.8 \pm 0.2^{\mathrm{a}}$ & $1.3 \pm 0.1^{\mathrm{b}}$ & $1.0 \pm 0.0^{\mathrm{cd}}$ & $0.8 \pm 0.1^{\mathrm{d}}$ & $4.0 \pm 0.0^{\mathrm{a}}$ \\
\hline$p$-Coumaric acid & $0.3 \pm 0.0$ & $0.2 \pm 0.0^{\mathrm{e}}$ & $0.3 \pm 0.0^{\mathrm{d}}$ & $0.3 \pm 0.0^{\mathrm{bcd}}$ & $0.4 \pm 0.0^{\mathrm{a}}$ & $0.1 \pm 0.0^{\mathrm{e}}$ & $0.4 \pm 0.0^{\text {abcd }}$ & $0.4 \pm 0.0$ abcd & $0.4 \pm 0.0 \mathrm{abc}$ & $0.3 \pm 0.1^{\mathrm{d}}$ & $0.3 \pm 0.0^{\mathrm{cd}}$ & $0.4 \pm 0.0 \mathrm{abcd}$ & $0.4 \pm 0.0 \mathrm{ab}$ \\
\hline Ferulic acid & $0.3 \pm 0.0$ & $0.2 \pm 0.0^{b}$ & $0.4 \pm 0.0^{\mathrm{a}}$ & $0.4 \pm 0.0^{\mathrm{a}}$ & $0.1 \pm 0.0^{c}$ & $0.2 \pm 0.0^{c}$ & $0.4 \pm 0.0^{\mathrm{a}}$ & $0.4 \pm 0.0^{\mathrm{a}}$ & $0.1 \pm 0.0^{c}$ & $0.4 \pm 0.0^{a}$ & $0.4 \pm 0.0^{\mathrm{a}}$ & $0.4 \pm 0.1^{\mathrm{a}}$ & $0.1 \pm 0.0^{c}$ \\
\hline $\begin{array}{c}\Sigma \text { Phenolic acids } \\
\text { Stilbenes }\end{array}$ & 62.2 & 19.7 & 77.2 & 72.1 & 72.8 & 19.7 & 78.7 & 71.9 & 74.2 & 77.0 & 77.0 & 70.3 & 75.4 \\
\hline $\begin{array}{l}\text { Stibenes } \\
\text { trans Resveratrol }\end{array}$ & $\mathrm{Nd}$ & $\mathrm{Nd}$ & $\mathrm{Nd}$ & $\mathrm{Nd}$ & $\mathrm{Nd}$ & $\mathrm{Nd}$ & $\mathrm{Nd}$ & $\mathrm{Nd}$ & $\mathrm{Nd}$ & $\mathrm{Nd}$ & $\mathrm{Nd}$ & $\mathrm{Nd}$ & $\mathrm{Nd}$ \\
\hline cis Resveratrol & $\mathrm{Nd}$ & $\mathrm{Nd}$ & $\mathrm{Nd}$ & $\mathrm{Nd}$ & $\mathrm{Nd}$ & $\mathrm{Nd}$ & $\mathrm{Nd}$ & $\mathrm{Nd}$ & $\mathrm{Nd}$ & $\mathrm{Nd}$ & $\mathrm{Nd}$ & $\mathrm{Nd}$ & $\mathrm{Nd}$ \\
\hline Piceatannol & $0.1 \pm 0.0$ & $0.2 \pm 0.0^{c}$ & $0.3 \pm 0.0^{b}$ & $0.3 \pm 0.0^{b}$ & $0.5 \pm 0.0^{\mathrm{a}}$ & $0.2 \pm 0.0^{c}$ & $0.3 \pm 0.0^{b}$ & $0.3 \pm 0.0^{b}$ & $0.5 \pm 0.0^{\mathrm{a}}$ & $0.3 \pm 0.0^{b}$ & $0.3 \pm 0.0^{\mathrm{b}}$ & $0.3 \pm 0.0^{b}$ & $0.5 \pm 0.0^{\mathrm{a}}$ \\
\hline Viniferin & $0.2 \pm 0.0$ & $0.2 \pm 0.0^{b}$ & $0.2 \pm 0.0^{\mathrm{a}}$ & $0.2 \pm 0.0^{\mathrm{a}}$ & $0.0 \pm 0.0^{\mathrm{c}}$ & $0.2 \pm 0.0^{\mathrm{b}}$ & $0.2 \pm 0.0^{\mathrm{a}}$ & $0.2 \pm 0.0^{\mathrm{a}}$ & $0.0 \pm 0.0^{\mathrm{c}}$ & $0.2 \pm 0.0^{\mathrm{a}}$ & $0.2 \pm 0.0^{\mathrm{a}}$ & $0.2 \pm 0.0^{\mathrm{a}}$ & $0.0 \pm 0.0^{\mathrm{c}}$ \\
\hline$\Sigma$ Stilbenes & 0.3 & 0.4 & 0.5 & 0.5 & 0.5 & 0.4 & 0.5 & 0.5 & 0.5 & 0.5 & 0.5 & 0.5 & 0.5 \\
\hline Total Phenolics $¥$ & $280.0 \pm 8.1$ & $245.0 \pm 18.1 \mathrm{abc}$ & $252.9 \pm 12.7^{\mathrm{ab}}$ & $231.4 \pm 5.4^{\mathrm{bcd}}$ & $214.7 \pm 8.3^{\mathrm{cd}}$ & $237.9 \pm 9.8 \mathrm{abcd}$ & $229.9 \pm 6.7^{\mathrm{bcd}}$ & $248.2 \pm 5.6^{\mathrm{ab}}$ & $150.0 \pm 1.1^{\mathrm{e}}$ & $212.5 \pm 20.5^{\mathrm{d}}$ & $212.3 \pm 6.7^{\mathrm{d}}$ & $267.0 \pm 1.9^{\mathrm{a}}$ & $225.2 \pm 11.2^{\mathrm{bcd}}$ \\
\hline
\end{tabular}

Averages followed by the same letters on a line do not differ between themselves according to the Tukey test $(p<0.05)$. Nd $=$ Not detected. ${ }^{¥}$ Total phenolics quantified through the

colorimetric method with Folin-Ciocalteu reagent. 
Table 4. Evolution of phenolic compounds $\left(\mathrm{mg} \mathrm{L}^{-1}\right)$ (mean \pm standard deviation) in the Syrah red wine stored in bottles of different colors for 12 months.

\begin{tabular}{|c|c|c|c|c|c|c|c|c|c|c|c|c|c|}
\hline \multirow{2}{*}{ Compounds } & \multirow{2}{*}{$\begin{array}{l}\text { Reference } \\
\text { (0 Months) }\end{array}$} & \multicolumn{4}{|c|}{ Clear } & \multicolumn{4}{|c|}{ Green } & \multicolumn{4}{|c|}{ Amber } \\
\hline & & 3 Months & 6 Months & 9 Months & 12 Months & 3 Months & 6 Months & 9 Months & 12 Months & 3 Months & 6 Months & 9 Months & 12 Months \\
\hline \multicolumn{14}{|l|}{ Flavonols } \\
\hline Kaempferol & $1.2 \pm 0.1$ & $1.1 \pm 0.0^{\mathrm{a}}$ & $1.0 \pm 0.0 \mathrm{abc}$ & $1.1 \pm 0.0^{\mathrm{a}}$ & $0.9 \pm 0.1^{\mathrm{cd}}$ & $1.0 \pm 0.1 \mathrm{abc}$ & $1.0 \pm 0.0 \mathrm{abc}$ & $1.1 \pm 0.0^{\mathrm{a}}$ & $0.9 \pm 0.0 \mathrm{bcd}$ & $1.1 \pm 0.0 \mathrm{ab}$ & $1.0 \pm 0.0 \mathrm{abc}$ & $1.0 \pm 0.1 \mathrm{abcd}$ & $0.9 \pm 0.1^{\mathrm{d}}$ \\
\hline Quercetin & $32.5 \pm 1.4$ & $23.2 \pm 0.1 \mathrm{ab}$ & $20.0 \pm 0.4^{\mathrm{c}}$ & $20.3 \pm 0.3^{\mathrm{c}}$ & $21.3 \pm 1.2^{b c}$ & $24.4 \pm 1.0^{\mathrm{a}}$ & $20.8 \pm 0.3^{b c}$ & $19.6 \pm 0.7^{c}$ & $21.2 \pm 1.1^{b c}$ & $24.3 \pm 1.0^{\mathrm{a}}$ & $20.6 \pm 0.8 \mathrm{bc}$ & $19.1 \pm 0.6^{c}$ & $21.0 \pm 1.7^{b c}$ \\
\hline Isorhamnetin & $4.1 \pm 0.3$ & $3.3 \pm 0.2^{\mathrm{a}}$ & $2.8 \pm 0.1^{\mathrm{b}}$ & $3.1 \pm 0.1^{\mathrm{b}}$ & $4.9 \pm 0.3^{\mathrm{b}}$ & $3.4 \pm 0.1^{\mathrm{a}}$ & $3.0 \pm 0.0^{\mathrm{b}}$ & $3.0 \pm 0.0^{\mathrm{b}}$ & $5.0 \pm 0.3^{\mathrm{b}}$ & $3.3 \pm 0.0^{\mathrm{a}}$ & $3.0 \pm 0.1^{\mathrm{b}}$ & $3.0 \pm 0.1^{\mathrm{b}}$ & $4.9 \pm 0.4^{\mathrm{b}}$ \\
\hline Myricetin & $1.0 \pm 0.0$ & $1.0 \pm 0.0^{\mathrm{a}}$ & $0.9 \pm 0.0^{\mathrm{b}}$ & $0.8 \pm 0.0^{c}$ & $0.9 \pm 0.0^{b c}$ & $1.0 \pm 0.0^{\mathrm{a}}$ & $0.9 \pm 0.0^{\mathrm{b}}$ & $0.8 \pm 0.0^{c}$ & $0.9 \pm 0.0^{\mathrm{bc}}$ & $1.0 \pm 0.0^{\mathrm{a}}$ & $0.9 \pm 0.0^{\mathrm{b}}$ & $0.9 \pm 0.0^{\mathrm{bc}}$ & $0.9 \pm 0.0^{\mathrm{bc}}$ \\
\hline Rutin & $1.7 \pm 0.2$ & $1.3 \pm 0.3^{c}$ & $1.7 \pm 0.1 \mathrm{abc}$ & $2.6 \pm 0.2^{\mathrm{ab}}$ & $2.9 \pm 0.7^{\mathrm{a}}$ & $1.6 \pm 0.2^{\mathrm{bc}}$ & $1.9 \pm 0.0 \mathrm{abc}$ & $2.2 \pm 0.5 \mathrm{abc}$ & $2.5 \pm 0.6 \mathrm{ab}$ & $1.8 \pm 0.1 \mathrm{abc}$ & $2.0 \pm 0.0 \mathrm{abc}$ & $1.9 \pm 0.1 \mathrm{abc}$ & $2.6 \pm 0.8 \mathrm{ab}$ \\
\hline$\Sigma$ Flavonols & 40.4 & 29.7 & 26.5 & 27.9 & 30.9 & 31.4 & 27.6 & 26.7 & 30.5 & 31.3 & 27.5 & 25.8 & 30.2 \\
\hline $\begin{array}{l}\text { Flavanols } \\
\text { Catechin }\end{array}$ & $26.1 \pm 1.9$ & $19.8 \pm 0.5 \mathrm{ab}$ & $17.5 \pm 0.9^{c}$ & $17.3 \pm 0.7^{\mathrm{c}}$ & $3.5 \pm 0.1^{\mathrm{e}}$ & $21.0 \pm 0.7^{a}$ & $18.7 \pm 0.0 \mathrm{bc}$ & & & & & & \\
\hline Epicatechin & $19.5 \pm 1.3$ & $13.4 \pm 1.0^{\mathrm{ab}}$ & $11.3 \pm 0.7^{\mathrm{cd}}$ & $10.7 \pm 0.3 \mathrm{de}$ & $1.7 \pm 0.1^{\mathrm{f}}$ & $13.9 \pm 0.4 \mathrm{a}$ & $12.2 \pm 0.4^{\mathrm{bc}}$ & $\begin{array}{l}11.4 \pm 0.2^{2} \\
10.6 \pm 0 . \mathrm{de}^{2}\end{array}$ & $\begin{array}{l}3.4 \pm 0.1^{\mathrm{e}} \\
1.6 \pm 0.1^{\mathrm{f}}\end{array}$ & $\begin{array}{l}20.5 \pm 0.3^{\mathrm{a}} \\
13.6 \pm 0.2^{\mathrm{ab}}\end{array}$ & $\begin{array}{l}18.4 \pm 0.8^{\mathrm{bc}} \\
11.7 \pm 0.7^{\mathrm{cd}}\end{array}$ & $\begin{array}{l}15.6 \pm 0.7^{\mathrm{a}} \\
9.8 \pm 0 . \mathrm{e}^{\mathrm{a}}\end{array}$ & $3.3 \pm 0.1^{\mathrm{e}}$ \\
\hline Epigallocatechin & $4.6 \pm 0.5$ & $2.2 \pm 0.0 \mathrm{def}$ & $3.9 \pm 0.1^{\mathrm{a}}$ & $3.7 \pm 0.3^{\mathrm{a}}$ & $1.4 \pm 0.0^{\mathrm{f}}$ & $3.1 \pm 0.0 \mathrm{abcd}$ & $3.5 \pm 0.2 \mathrm{ab}$ & $2.6 \pm 0.9$ bcde & $1.7 \pm 0.1^{\text {ef }}$ & $\begin{array}{l}13.6 \pm 0.2 \mathrm{ab} \\
3.4 \pm 0.1 \mathrm{a}\end{array}$ & $\begin{array}{l}11.7 \pm 0.8 \\
3.3 \pm 0.8 \mathrm{abc}\end{array}$ & $\begin{array}{l}9.8 \pm 0.6 \\
2.3 \pm 0.1 \text { def }\end{array}$ & $\begin{array}{l}1.5 \pm 0.1^{\mathrm{i}} \\
1.7 \pm 0 . \mathrm{f}^{\mathrm{n}}\end{array}$ \\
\hline Epicatechin gallate & $3.2 \pm 0.8$ & $2.8 \pm 0.2^{b}$ & $2.6 \pm 0.1^{\mathrm{b}}$ & $2.7 \pm 0.1^{\mathrm{b}}$ & $3.4 \pm 0.3^{\mathrm{a}}$ & $2.9 \pm 0.1 \mathrm{~b}$ & $2.7 \pm 0.1^{b}$ & $2.6 \pm 0.1^{b}$ & $2.9 \pm 0.2^{\mathrm{b}}$ & $2.7 \pm 0.1^{\mathrm{b}}$ & $2.6 \pm 0.2^{b}$ & $2.5 \pm 0.1^{\mathrm{b}}$ & $2.9 \pm 0.1^{\mathrm{b}}$ \\
\hline$\Sigma$ Flavanols & 53.3 & 40,2 & 35.4 & 36.3 & 9.6 & 41 & 37 & 33.1 & 9.7 & 40.2 & 2.010 .2 & 30.1 & 9.4 \\
\hline \multicolumn{14}{|l|}{ Proanthocyanidins } \\
\hline $\begin{array}{l}\text { Procyanidin A2 } \\
\text { Procyanidin B1 }\end{array}$ & $\begin{array}{l}4.2 \pm 0.1 \\
18.8+04\end{array}$ & $\begin{array}{l}3.5 \pm 0.0 \text { a dac } \\
21.1 \pm 0.3\end{array}$ & $\begin{array}{l}3.1 \pm 0.0 \mathrm{c} \\
21.3+0.5 \mathrm{a}\end{array}$ & $\begin{array}{l}2.9 \pm 0.2^{2} \\
21.5 \pm 0.7 \mathrm{a}\end{array}$ & $\begin{array}{l}1.0 \pm 0.0 \\
36.8+0.8^{b}\end{array}$ & $\begin{array}{l}3.6 \pm 0.1 \mathrm{a} \\
22.4+0.5 \mathrm{a}\end{array}$ & $3.3 \pm 0.0^{\mathrm{ccd}}$ & $3.1 \pm 0.3 \mathrm{de}$ & $1.0 \pm 0.0^{\mathrm{f}}$ & $3.6 \pm 0.1^{\mathrm{ab}}$ & $3.2 \pm 0.1^{\mathrm{cd}}$ & $2.9 \pm 0.1^{\mathrm{e}}$ & $1.0 \pm 0.0^{\mathrm{f}}$ \\
\hline $\begin{array}{l}\text { Procyanidin B1 } \\
\text { Procyanidin B2 }\end{array}$ & $18.8 \pm 0.4$ & & for & $254+09 c$ & & $22.4 \pm 0.5^{\mathrm{a}}$ & $22.6 \pm 0.4^{\mathrm{a}}$ & $21.6 \pm 0.2^{\mathrm{a}}$ & $39.0 \pm 0.2^{\mathrm{a}}$ & $21.6 \pm 0.2^{\mathrm{a}}$ & $21.4 \pm 1.5^{\mathrm{a}}$ & $18.7 \pm 0.3^{\mathrm{d}}$ & $37.3 \pm 0.2 \mathrm{ab}$ \\
\hline $\begin{array}{l}\text { Procyanidin B2 } \\
\text { Proynthoniding }\end{array}$ & $21.7 \pm 0.5$ & $22.0 \pm 1.1 \mathrm{de}$ & $19.5 \pm 0.4^{\mathrm{f}}$ & $25.4 \pm 0.9^{\mathrm{c}}$ & $30.8 \pm 1.0^{\mathrm{b}}$ & $22.4 \pm 0.5$ de & $21.9 \pm 0.6^{\text {def }}$ & $24.3 \pm 1.5^{\mathrm{cd}}$ & $33.4 \pm 0.5^{\mathrm{a}}$ & $21.9 \pm 0.5 \operatorname{def}$ & $21.4 \pm 0.5$ ef $^{-1}$ & $20.7 \pm 1.4^{\text {ef }}$ & $31.9 \pm 0.1 \mathrm{ab}$ \\
\hline \multicolumn{14}{|l|}{$\begin{array}{l}\Sigma \text { Proanthocyanidins } \\
\text { Phenolic acids }\end{array}$} \\
\hline $\begin{array}{l}\text { Phenolic acids } \\
\text { Gallic acid }\end{array}$ & $25.0 \pm 1.4$ & $29.1 \pm 0.8^{\mathrm{e}}$ & $30.9 \pm 0.9$ cde & $33.9 \pm 0.7^{\mathrm{a}}$ & $33.5 \pm 0.6 \mathrm{ab}$ & $29.7 \pm 1.0 \mathrm{de}$ & $31.57 .6 \pm 0.2^{\mathrm{bcd}}$ & $33.8 \pm 0.4^{\mathrm{a}}$ & $33.6 \pm 0.4^{\mathrm{a}}$ & $28.9 \pm 0.6^{\mathrm{e}}$ & $31.4 \pm 0.8^{\mathrm{cd}}$ & $32.1 \pm 0.1 \mathrm{abc}$ & $31.8 \pm 1.9 \mathrm{abc}$ \\
\hline Caffeic acid & $9.6 \pm 0.3$ & $9.1 \pm 0.0^{\mathrm{bc}}$ & $9.1 \pm 0.4^{\mathrm{bc}}$ & $8.5 \pm 0.1$ & & $10.6 \pm 0.2^{\mathrm{a}}$ & $9.4 \pm 0.3^{\mathrm{bc}}$ & $9.5 \pm 0$ & $8.6=$ & $9.9 \pm 0.5^{\mathrm{ab}}$ & & & $8.6 \pm 0.8^{\mathrm{cd}}$ \\
\hline Caftaric acid & $91.2 \pm 0.7$ & $84.6 \pm 1.2 \mathrm{ab}$ & $77.3 \pm 2.6$ cde & $76.4 \pm 0.3$ de & $75.4 \pm 1.1^{\mathrm{e}}$ & $85.6 \pm 1.0^{\mathrm{a}}$ & $80.8 \pm 1.6^{b c}$ & $77.4 \pm 0.5$ cde & $76.6 \pm 1.1 \mathrm{de}$ & $86.8 \pm 2.3^{a}$ & $79.6 \pm 1.6^{\mathrm{cd}}$ & $77.6 \pm$ & $76.1 \pm 0.6$ de \\
\hline Chlorogenic acid & $4.4 \pm 0.3$ & $4.4 \pm 0.1^{\mathrm{b}}$ & $5.7 \pm 0.2^{\mathrm{a}}$ & $5.6 \pm 0.3^{\mathrm{a}}$ & $5.5 \pm 0.2^{\mathrm{a}}$ & $5.8 \pm 0.1^{\mathrm{a}}$ & $5.6 \pm 0.1^{\mathrm{a}}$ & $6.1 \pm 0.2^{\mathrm{a}}$ & $5.7 \pm 0.6^{\mathrm{a}}$ & $5.3 \pm 0.5^{\mathrm{a}}$ & $5.8 \pm 0.3^{a}$ & $5.6 \pm 0.2^{\mathrm{a}}$ & $5.9 \pm 0.5 \mathrm{a}$ \\
\hline$p$-Coumaric acid & $1.4 \pm 0.0$ & $2.4 \pm 0.0^{\mathrm{de}}$ & $2.3 \pm 0.0^{\mathrm{de}}$ & $3.2 \pm 0.0^{\mathrm{b}}$ & $3.8 \pm 0.0^{\mathrm{a}}$ & $2.3 \pm 0.0^{\mathrm{e}}$ & $2.4 \pm 0.0^{\mathrm{de}}$ & $3.1 \pm 0.1 \mathrm{bc}$ & $3.7 \pm 0.2^{\mathrm{a}}$ & $1.9 \pm 0.1^{\mathrm{f}}$ & $2.5 \pm 0.0^{\mathrm{d}}$ & $3.0 \pm 0.0^{c}$ & $3.8 \pm 0.1^{\mathrm{a}}$ \\
\hline Ferulic acid & $3.1 \pm 0.1$ & $2.8 \pm 0.0^{\mathrm{a}}$ & $2.5 \pm 0.1^{\mathrm{b}}$ & $2.5 \pm 0.1^{\mathrm{b}}$ & $2.3 \pm 0.1^{\mathrm{c}}$ & $2.8 \pm 0.1^{\mathrm{a}}$ & $2.6 \pm 0.0^{\mathrm{b}}$ & $2.6 \pm 0.0^{\mathrm{b}}$ & $2.3 \pm 0.1^{c}$ & $2.8 \pm 0.1^{\mathrm{a}}$ & $2.6 \pm 0.1^{\mathrm{b}}$ & $2.6 \pm 0.1^{\mathrm{b}}$ & $2.2 \pm 0.1^{\mathrm{c}}$ \\
\hline $\begin{array}{l}\Sigma \text { Phenolic acids } \\
\text { Stilbenes }\end{array}$ & 134.6 & 132.5 & 127.8 & 130.0 & 128.4 & 136.9 & 132.4 & 132.5 & 130.5 & 135.7 & 131.2 & 130.2 & 128.4 \\
\hline trans Resveratrol & $0.6 \pm 0.0$ & $0.6 \pm 0.0^{\mathrm{ab}}$ & $0.6 \pm 0.0^{\mathrm{ab}}$ & $0.6 \pm 0.0^{\mathrm{bc}}$ & $0.5 \pm 0.0^{\mathrm{c}}$ & $0.6 \pm 0.0^{\mathrm{a}}$ & $0.6 \pm 0.0 \mathrm{ab}$ & $0.6 \pm 0.1 \mathrm{ab}$ & $0.5 \pm 0.0^{c}$ & $0.6 \pm 0.0^{\mathrm{ab}}$ & $0.6 \pm 0.0^{\mathrm{ab}}$ & $0.6 \pm 0.0 \mathrm{ab}$ & $0.5 \pm 0.0^{\mathrm{c}}$ \\
\hline cis Resveratrol & $0.8 \pm 0.1$ & $0.4 \pm 0.0^{\mathrm{cd}}$ & $0.5 \pm 0.1 \mathrm{ab}$ & $0.4 \pm 0.0^{\mathrm{cd}}$ & $0.6 \pm 0.0^{\mathrm{a}}$ & $0.4 \pm 0.0^{\mathrm{cd}}$ & $0.4 \pm 0.0^{\mathrm{bcd}}$ & $0.5 \pm 0.0^{b c}$ & $0.6 \pm c$ & $0.4 \pm 0.0^{\mathrm{cd}}$ & $0.4 \pm 0.0^{\mathrm{d}}$ & $0.4 \pm 0.0^{\mathrm{cd}}$ & $0.5 \pm 0.0^{\mathrm{a}}$ \\
\hline Piceatannol & $2.0 \pm 0.1$ & $1.6 \pm 0.0^{\mathrm{bc}}$ & $1.4 \pm 0.0 \mathrm{de}$ & $1.4 \pm 0.1^{\mathrm{d}}$ & $0.8 \pm 0.0^{\mathrm{f}}$ & $1.7 \pm 0.1^{\mathrm{a}}$ & $1.5 \pm 0.0^{\mathrm{cd}}$ & $1.4 \pm 0.1^{\mathrm{d}}$ & $0.8 \pm 0.0^{\mathrm{f}}$ & $1.7 \pm 0.0$ ab & $1.5 \pm 0.1^{\mathrm{cd}}$ & $1.3 \pm 0.0^{\mathrm{e}}$ & $0.8 \pm 0.0^{\mathrm{f}}$ \\
\hline Viniferin & $0.8 \pm 0.0$ & $0.8 \pm 0.0^{\mathrm{ab}}$ & $0.8 \pm 0.0^{c}$ & $0.8 \pm 0.0^{c}$ & $0.7 \pm 0.0^{\mathrm{d}}$ & $0.8 \pm 0.0^{\mathrm{a}}$ & $0.8 \pm 0.0^{\mathrm{cd}}$ & $0.8 \pm 0.0^{\text {cd }}$ & $0.7 \pm 0.0^{\mathrm{d}}$ & $0.8 \pm 0.0^{\mathrm{cd}}$ & $0.8 \pm 0.0^{\text {cd }}$ & $0.8 \pm 0.0^{c}$ & $0.7 \pm 0.0^{\mathrm{d}}$ \\
\hline$\sum$ Stilbenes & 4.2 & 3.4 & 3.3 & 3.2 & 2.6 & 3.5 & 3.3 & 3.3 & 2.6 & 3.5 & 3.3 & 3.1 & 2.6 \\
\hline $\begin{array}{l}\text { Anthocyanins } \\
\text { Pelargonidin }\end{array}$ & $15.8 \pm 0.4$ & $9.1 \pm 0.4^{\mathrm{a}}$ & $5.3 \pm 0.5^{b}$ & $4.1 \pm 0.2^{\mathrm{c}}$ & $2.9 \pm 0.2^{\mathrm{d}}$ & $9.8 \pm 0.3^{\mathrm{a}}$ & $6.1 \pm 0.2^{b}$ & $4.0 \pm 0.1^{\mathrm{cd}}$ & $3.4 \pm 0.1^{\mathrm{cd}}$ & $9.2 \pm 0.6^{\mathrm{a}}$ & $5.7 \pm 0.6^{\mathrm{b}}$ & $3.7 \pm 0.7^{\mathrm{cd}}$ & $3.2 \pm 0.1^{\mathrm{cd}}$ \\
\hline $\begin{array}{c}\text { 3-glucoside } \\
\text { Cyanidin 3-glucoside }\end{array}$ & $1.2 \pm 0.0$ & $1.1 \pm 0.0^{\mathrm{a}}$ & $0.8 \pm 0.0^{\mathrm{de}}$ & $0.7 \pm 0.0$ efg & $0.6 \pm 0.0 \mathrm{~g}$ & $1.0 \pm 0.1 \mathrm{ab}$ & $0.8 \pm 0.1^{\mathrm{cd}}$ & $0.7 \pm 0.0 \operatorname{defg}$ & $0.6 \pm 0.0^{\mathrm{g}}$ & $0.9 \pm 0.1^{b c}$ & $0.8 \pm 0.0$ def & $0.7 \pm 0.0^{\mathrm{fg}}$ & $0.6 \pm 0.0 \mathrm{~g}$ \\
\hline $\begin{array}{l}\text { Delphinidin } \\
\text { 3-glucoside }\end{array}$ & $9.9 \pm 0.2$ & $6.3 \pm 0.2^{\mathrm{a}}$ & $3.8 \pm 0.4^{\mathrm{cd}}$ & $3.26 \pm 0.07 \mathrm{de}$ & $2.1 \pm 0.1 \mathrm{~g}$ & $6.7 \pm 0.2^{\mathrm{a}}$ & $4.5 \pm 0.2^{b}$ & $3.1 \pm 0.0^{\mathrm{e}}$ & $2.4 \pm 0.1^{\mathrm{fg}}$ & $6.3 \pm 0.2^{\mathrm{a}}$ & $4.2 \pm 0.4 \mathrm{bc}$ & $2.9 \pm 0.4^{\mathrm{ef}}$ & $2.3 \pm 0.1 \mathrm{fg}$ \\
\hline $\begin{array}{l}\text { 3-gucosidee } \\
\text { Malvidin 3-glucoside }\end{array}$ & $88.5 \pm 1.6$ & $52.4 \pm 0.8^{\mathrm{a}}$ & $29.7 \pm 3.0^{\mathrm{c}}$ & $22.7 \pm 0.8^{\mathrm{d}}$ & $13.6 \pm 0.0^{\mathrm{f}}$ & $55.7 \pm 1.0^{\mathrm{a}}$ & $34.6 \pm 1.4^{\mathrm{b}}$ & $22.0 \pm 0.1^{\mathrm{d}}$ & $16.6 \pm 0.5^{\text {ef }}$ & $55.0 \pm 1.8^{\mathrm{a}}$ & $32.3 \pm 2.5^{b c}$ & $17.9 \pm 0.5^{\mathrm{e}}$ & $15.6 \pm 0.4^{\text {ef }}$ \\
\hline Peonidin 3-glucoside & $14.2 \pm 0.7$ & $8.8 \pm 0.3^{a}$ & $4.8 \pm 0.6^{\mathrm{b}}$ & $3.6 \pm 0.1^{\mathrm{c}}$ & $2.5 \pm 0.2^{\mathrm{d}}$ & $8.8 \pm 0.2^{\mathrm{a}}$ & $5.7 \pm 0.4^{\mathrm{b}}$ & $3.5 \pm 0.1^{\mathrm{cd}}$ & $2.8 \pm 0.1^{\mathrm{cd}}$ & $8.7 \pm 0.3^{\mathrm{a}}$ & $5.2 \pm 0.5^{\mathrm{b}}$ & $3.2 \pm 0.5^{\mathrm{cd}}$ & $2.7 \pm 0.1^{\mathrm{cd}}$ \\
\hline Petunidin 3-glucoside & $4.3 \pm 0.8$ & $2.3 \pm 0.1^{\mathrm{a}}$ & $1.4 \pm 0.1^{\mathrm{c}}$ & $1.32 \pm 0.01^{\mathrm{c}}$ & $1.0 \pm 0.0^{\mathrm{d}}$ & $2.5 \pm 0.1^{\mathrm{a}}$ & $1.6 \pm 0.0^{\mathrm{b}}$ & $1.3 \pm 0.0^{c}$ & $1.1 \pm 0.0^{\mathrm{d}}$ & $2.4 \pm 0.1^{\mathrm{a}}$ & $1.5 \pm 0.0 \mathrm{bc}$ & $1.1 \pm 0.1^{\mathrm{d}}$ & $1.1 \pm 0.0^{\mathrm{d}}$ \\
\hline $\begin{array}{l}\sum \text { Anthocyanins } \\
\text { Total Monomeric }\end{array}$ & 134.4 & 80.5 & 45.7 & (1) & 22.7 & 84.5 & 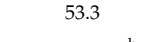 & 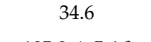 & 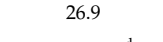 & (n) & & 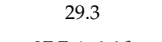 & 26.4 \\
\hline $\begin{array}{l}\text { Total Monomeric } \\
\text { Anthocyanins }\end{array}$ & $243.6 \pm 1.0$ & $179.0 \pm 3.0^{\mathrm{a}}$ & $129.0 \pm 6.2^{\mathrm{b}}$ & $94.2 \pm 1.0^{\mathrm{c}}$ & $38.2 \pm 1.0^{\mathrm{e}}$ & $182.6 \pm 5.0^{\mathrm{a}}$ & $135.1 \pm 5.4^{\mathrm{b}}$ & $105.8 \pm 5.4^{c}$ & $65.8 \pm 2.0^{\mathrm{d}}$ & $186.3 \pm 4.9^{\mathrm{a}}$ & $126.6 \pm 3.0^{\mathrm{b}}$ & $97.7 \pm 6.6^{c}$ & $73.8 \pm 1.0^{\mathrm{d}}$ \\
\hline Total Phenolics * & $2439.8 \pm 22.0$ & $1945.6 \pm 86.3^{\mathrm{d}}$ & $1955.3 \pm 62.3^{\mathrm{cd}}$ & $2125.2 \pm 27.9 \mathrm{abc}$ & $1933.2 \pm 37.6^{\mathrm{d}}$ & $2023.9 \pm 8.0 \mathrm{bcd}$ & $2050.9 \pm 9.3 \mathrm{bcd}$ & $2236.7 \pm 18.6^{\mathrm{a}}$ & $2029.2 \pm 93.1 \mathrm{bcd}$ & $1954.6 \pm 58.5^{\mathrm{cd}}$ & $2071.8 \pm 24.1 \mathrm{abcd}$ & $2193.4 \pm 51.2^{\mathrm{ab}}$ & $2094.3 \pm 115.4$ abcd \\
\hline
\end{tabular}

Averages followed by the same letters on a line do not differ between themselves according to the Tukey test $(p<0.05) .{ }^{¥}$ Total phenolics quantified through the colorimetric method with Folin-Ciocalteu reagent. ${ }^{\Psi}$ Total monomeric anthocyanins quantified through the $\mathrm{pH}$ differential method. 


\subsubsection{Sparkling Moscatel}

The profiles for the evolution of the phenolic compounds during the storage of the sparkling Moscatel wine in the bottles with different colors did not show considerable changes in the flavanols, phenolic acids and stilbenes during the storage time studied (Table 3). However, the flavanol catechin showed a significant increase during storage and this increase was similar in bottles of all colors. According to Garrido and Borges [23] changes in polyphenolic composition are due to the participation of grape phenolics in haze formation and in numerous copigmentation, cycloaddition, polymerization, hydrolysis, and oxidation reactions. These enzymatic and non-enzymatic reactions start just after grape crushing and continue throughout fermentation and aging, contributing to important changes in the wine sensory properties and to the obtention of a great diversity of new phenolic structures. Previous studies conducted by Maury et al. [9], Clark et al. [7], Dias et al. [12] and Xing et al. [24] also evidenced an increase in the catechin contents during the aging process of white wines. This factor could explain the slight increase in the $+\mathrm{b}^{*}$ values (yellow color) for the sparkling Moscatel during storage (see Table 2 ), suggesting that aging had occurred.

\subsubsection{Syrah Red Wine}

In relation to the evolution of the phenolic compounds profile for the Syrah red wine (Table 4), the flavonols did not show notable changes during storage in the bottles of different colors. On the other hand, for the flavanol group, there was a decrease in the content of catechin and epicatechin. This behavior was also observed by Marquez et al. [10], who studied the stability of sweet Merlot, Syrah and Tempranillo red wines over 12 months. The decrease in the catechin and epicatechin levels could be due (tannin/tannin and/or tannin/anthocyanin) polymerization, or copigmentation occurring during the evolution of red wines [25].

These polymerizations and copigmentation reactions, in turn, lead to a change from an intense red color to an orange color [26], which explains the increase in the hue $\left(\mathrm{h}^{*}\right)$ observed for the Syrah wine after 12 months of storage (see Table 2).

In relation to the phenolic acids, caftaric acid was the major compound in the Syrah wine and it decreased during the storage time in each of the different-colored bottles of different colors (with values ranging from 91.2 to $75.4 \mathrm{mg} \mathrm{L}^{-1}$ ). This behavior was also observed in red wines by Scrimgeour, Nordestgaard, Lloyd, Wilkes [27], who attributed this decrease to this compound's high affinity for the oxygen dissolved in wines. This oxidation reaction can adversely affect the sensory quality of the wine [23].

In relation to the stilbenes, the concentration of piceatannol decreased in the bottles of all colors. This compound is considered important since, even in low concentrations, its consumption has been associated with a decrease in the incidence of cardiovascular diseases [28]. In the Syrah red wine of the $\mathrm{SFV}$, the concentration of piceatannol in recently produced wine was $2 \mathrm{mg} \mathrm{L}^{-1}$, and after 12 months of storage in bottles it was $0.76 \mathrm{mg} \mathrm{L}^{-1}$. Guerrero et al. [29] observed a piceatannol concentration of $1.05 \mathrm{mg} \mathrm{L}^{-1}$ in red wine produced from the Jaen grape variety, after applying artificial UV light to grapes in order to stimulate their synthesis, which suggests that the values found for the Syrah red wine of the SFV can be considered in a natural way.

During storage, in the bottles of all colors, there was a decrease of the total monomeric anthocyanins in the wine, as well as of the individual anthocyanins, as quantified through high-performance liquid chromatography (HPLC). Similar behavior has been observed in previous studies on red wine storage in bottles of a single color [10,30]. Specifically, during storage in clear bottles for 12 months the total monomeric anthocyanins decreased by $84.3 \%$ and this coincided with an $84.6 \%$ decrease in the content of malvidin 3-glucoside, which is the main anthocyanin responsible for the color of Vitis vinifera L. wines. These results suggest that the use of clear bottles may be detrimental to the preservation of the red wine color when the storage period exceeds a year. 


\subsection{Principal Component Analysis}

The Principal Components Analysis (PCA) was applied in the different treatments as a function of individual phenolic compounds and antioxidant activity. For the sparkling Moscatel (Figure 1A), the first two principal components (PC1 + PC2) explained $92.81 \%$ of the data variance. For analysis purposes, only compounds with a loading $>0.70$ was considered. The PC1 with the highest percentage of explained variance $(57.80 \%)$ grouped the treatments MGB3 and MCB3 in the negative part of the PC1 associated with the negative loading for myricetin, and positive for procyanidins A2 and B2. The PC2 with the lowest percentage of explained variance (35.01\%) grouped the wines with 6 and 9 months of bottle storage, in the positive part, associated with the loadings for ferulic acid, procyanidin A2 and B2, epicatechin gallate and viniferin. The wines with 12 months of storage were grouped in the negative part (PC2) with the compounds procyanidin B1, catechin and chlorogenic acid. Based on the PCA, the separations of treatments obtained for sparkling Moscatel were associated with the storage time.

In relation to the Syrah red wine (Figure 1B), the first two main components (PC1 + PC2) explained $93.8 \%$ of the data variance. The treatments with 3 months of storage in the bottles of the three colors (SCB3, SGB3 and SAB3) were grouped in the positive part of the PC2, and the compounds responsible for this separation were petunidin, quercetin, myricetin, caftaric acid and ferulic acid.
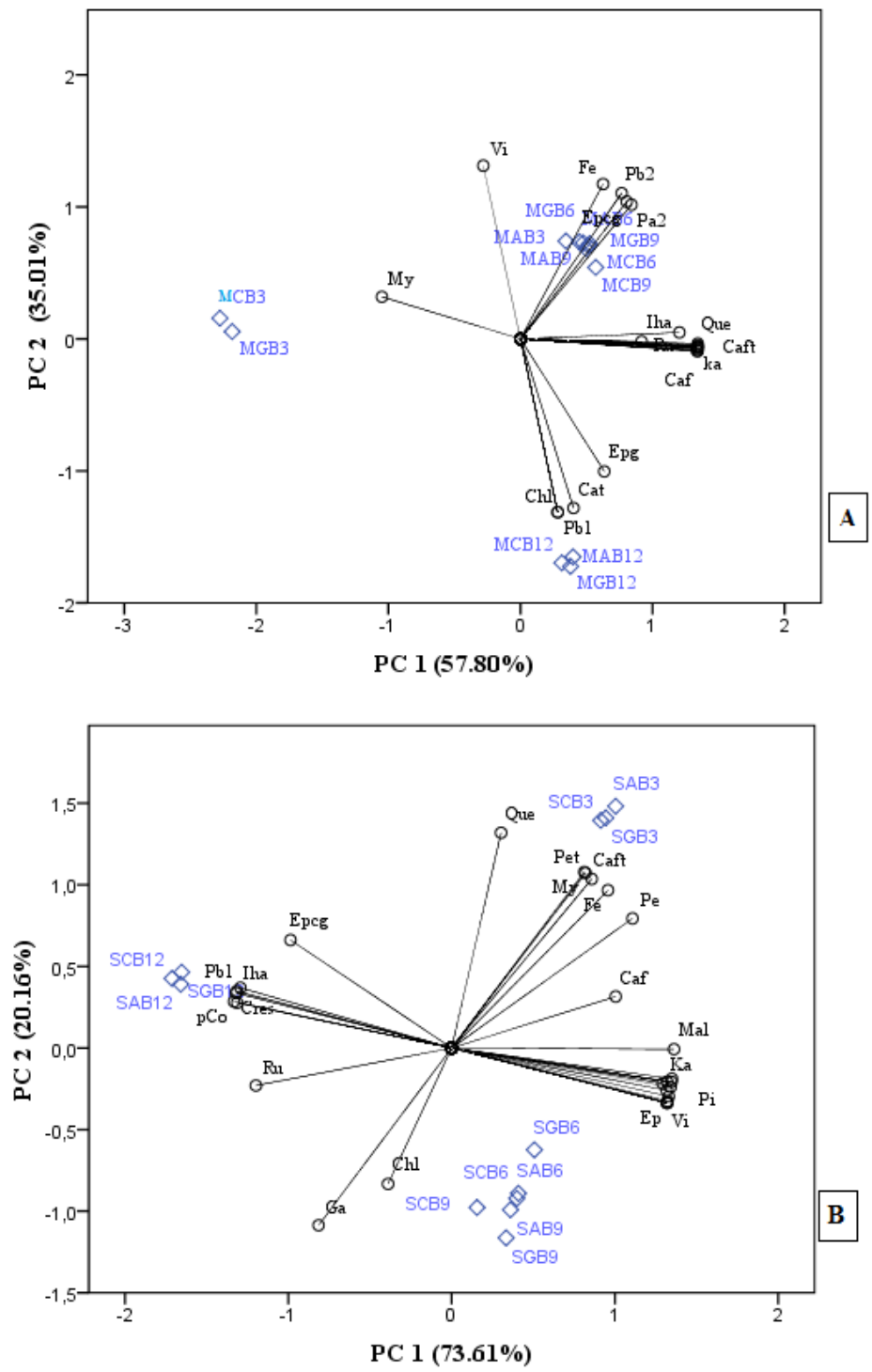

Figure 1. Principal component analysis (PCA) of phenolic compounds and the antioxidant activity results for sparkling Moscatel (A) and Syrah (B) wines stored in bottles of different colors for 12 months. 
Legend: clear bottle 3 months (CB3), green bottle 3 months (GB3), amber bottle 3 months (AB3); clear bottle 6 months (CB6), green bottle 6 months (GB6), amber bottle 6 months (AB6); clear bottle 9 months (CB9), green bottle 9 months (GB9), amber bottle 9 months (AB9); clear bottle 12 months (CB12), green bottle 12 months (GB12), amber bottle 12 months (AB12). Kaempferol 3-glucoside (ka); quercetin 3-glucoside (Que); isorhamnetin (Iha); myricetin (My); rutin (Ru); caffeic acid (Caf); caftaric acid (caft); chlorogenic acid (Chl); $p$-coumaric (p-Co); ferulic acid (Fe); piceatannol (Pi); catechin (Cat); epicatechin (Ep); procyanidin A2 (Pa2); procyanidin B1 (Pb1); procyanidin B2 (Pb2); gallic acid (Ga); epigallocatechin (Epg); epicatechin gallate (Epcg); viniferin (Vi); pelargonidin (Pel); cyanidin (Cy); delphinidin (Del); malvidin (Mal); peonidin (Pe); petunidin (Pet); trans-resveratrol (Ter); cis-resveratrol (Cre). $\diamond=$ treatments studied.

Bottles of all colors stored for 6 and 9 months (SCB6, SGB6, SAB6; SCB9, SGB9, and SAB9) were grouped in the negative part of the PC2, associated with chlorogenic acid and gallic acid. The treatments for 12 months in bottles of all colors (SCB12, SGB12, and SAB12) were grouped in the negative part of the PC1 and were associated with higher values for isorhamnetin, rutin, $p$-coumaric acid, procyanidin B1 and B2, epicatechin gallate and cis-resveratrol and lower values for malvidin 3-glucoside, catechin and epicatechin.

Overall, the PCA of the results for the two wines studied showed that the evolution of the profile for the phenolic compounds studied was due to the storage time of up to 12 months and not the color of the bottles used to store the wines.

\subsection{Antioxidant Activity In Vitro}

The results for the antioxidant activity (AOX) of the wines studied are given in Figure 2. The AOX for the sparkling Moscatel measured with the DPPH method (Figure 2A) showed an increase during sixth months of storage for the clear bottles and ninth months of storage for the green and amber bottles. In relation to the AOX measured through the ABTS method, the AOX increased in the wines with six months of storage in the green and amber bottles and nine months in the clear bottles. Kallithraka, Salacha, Tzourou [31] studied the aging of white wine and also observed an increase in the antioxidant activity after six months of bottle storage. Antioxidant activity is specific for each individual phenolic compound [32]. The enzymatic and non-enzymatic reactions during aging of wine contribute to important changes and attainment of new phenolic structures; which could explain the increase in AOX during storage [23].

In the case of the Syrah red wine, the AOX measured through both methods showed an increase as a function of the storage time for the amber bottles, while the wine stored in the clear and green bottles remained stable with regard to this. The AOX values obtained in this study are in agreement with those reported by Padilha et al. [33] for commercial Syrah red wines of the SFV, and they are considered to be high. In general, the wines stored in bottles of different colors showed stability with regard to the antioxidant activity in vitro, which is a positive result since the AOX of wines is normally associated with consumer health protection in relation to several diseases [34]. 

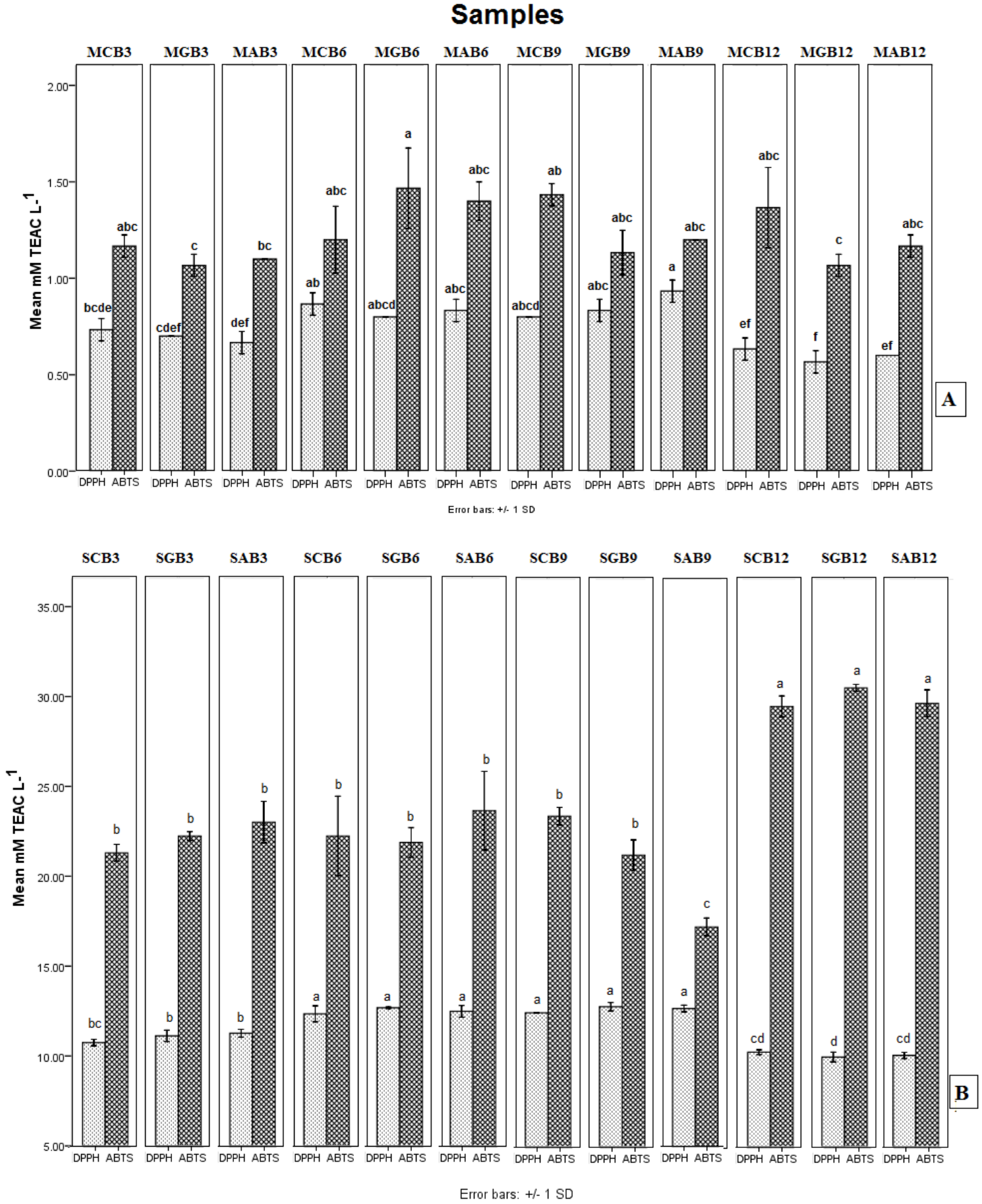

Figure 2. Antioxidant activity in vitro of the sparkling Moscatel (A) and Syrah (B) wines stored in bottles of different colors for 12 months. DPPH: 2,2-diphenyl-1-picrylhydrazyl; ABTS: 2,2'-azino-bis (3-ethylbenzothiazoline-6-sulfonic acid).

\section{Conclusions}

The different bottle colors studied did not influence the evolution of the sparkling Moscatel and Syrah red wines since the main variations observed were associated with the storage time. The main changes observed in the Syrah red wine were an increase in hue $\left(\mathrm{h}^{*}\right)$, a decrease in catechins, an increase in procyanidins and, most notably, a decrease in the anthocyanin malvidin 3-glucoside, which is the main compound responsible for the color of red wines produced from $V$. vinifera L. grapes. In general, the wines studied presented stability in terms of the antioxidant activity in vitro. 
Author Contributions: Conceptualization, J.F.d.S. and A.M.d.S.N.; Formal analysis, M.d.S.S.L. and M.d.C.P.D.; Writing and Methodology, M.d.S.L. and G.E.P.

Funding: CAPES (Coordination for the Improvement of Higher Education Personnel).

Acknowledgments: The authors would like to thank CAPES (Coordination for the Improvement of Higher Education Personnel) for the concession of scholarships and financial support. The authors would also like to thank the Miolo Wine Group, Piagentini and Botticelli for supplying the raw-material. The English text of this paper has been revised by Sidney Pratt, Canadian, MAT (The Johns Hopkins University), RSAdip-TESL (Cambridge University).

Conflicts of Interest: The author declares that there is no conflict of interests regarding the publication of this article.

\section{References}

1. Gomes, L.S.; José-Coutinho, A.; Silva, A.G.; Ricardo-da-Silva, J.M. Sensory profile characterization and typicality assessment of "bairrada" and "beira atlântico" red wines. Ciência e Técnica Vitivinícola 2016, 31, 73-87. [CrossRef]

2. Dutra, M.C.P.; De Souza, J.F.; Viana, A.C.; De Oliveira, D.; Pereira, G.E.; Dos Santos Lima, M. Rapid determination of the aromatic compounds methyl-anthranilate, 2 '-aminoacetophenone and furaneol by GC-MS: Method validation and characterization of grape derivatives. Food Res. Int. 2018, 107, 613-618. [CrossRef] [PubMed]

3. Pereira, G.E.; Padinha, C.; Biasoto, A.C.T.; Canuto, K.M.; Nascimento, M.A.S.; Souza, J.F. Le poids des consommateurs sur l'évolution des vins: L'exemple de la Vallée du São Francisco, Brésil. In Rencontres du Clos-VOugeot 2015: "Vin et civilisation. Les étapes de l'humanisation"; Pérard, J., Perrot, M., Eds.; Centre Georges Chevrier: Dijon, France, 2016; Volume 9, p. 301.

4. Ricci, A.; Parpinello, G.P.; Versari, A. Modelling the evolution of oxidative browning during storage of white wines: Effects of packaging and closures. Int. J. Food Sci. Technol. 2017, 52, 472-479. [CrossRef]

5. Agriopoulou, S.; Stamatelopoulou, E. Influence of Storage Conditions on the Quality Characteristics of Wines. EC Nutr. 2017, 8, 93-98.

6. Arapitsas, P.; Speri, G.; Angeli, A.; Perenzoni, D.; Mattivi, F. The influence of storage on the "chemical age" of red wines. Metabolomics 2014, 10, 816-832. [CrossRef]

7. Clark, A.C.; Dias, D.A.; Smith, T.A.; Ghiggino, K.P.; Scollary, G.R. Iron (III) Tartrate as a Potential Precursor of Light-Induced Oxidative Degradation of White Wine: Studies in a Model Wine System. J. Agric. Food Chem. 2011, 59, 3575-3581. [CrossRef] [PubMed]

8. Del Caro, A.; Piombino, P.; Genovese, A.; Moio, L.; Fanara, C.; Piga, A. Effect of Bottle Storage on Colour, Phenolics and Volatile Composition of Malvasia and Moscato White Wines. S. Afr. J. Enol. Vitic. 2014, 35, 128-138. [CrossRef]

9. Maury, C.; Clarkb, A.C.; Scollary, G.R. Determination of the impact of bottle colour and phenolic concentration on pigment development in white wine stored under external conditions. Anal. Chim. Acta 2010, 660, 81-86. [CrossRef] [PubMed]

10. Marquez, A.; Serratosa, M.P.; Merida, J. Influence of bottle storage time on colour, phenolic composition and sensory properties of sweet red wines. Food Chem. 2014, 146, 507-514. [CrossRef] [PubMed]

11. Dias, D.A.; Smith, T.A.; Ghiggino, K.P.; Scollary, G.R. The role of light, temperature and wine bottle colour on pigment enhancement in white wine. Food Chem. 2012, 135, 2934-2941. [CrossRef] [PubMed]

12. Dias, D.A.; Clark, A.C.; Smith, T.A.; Ghiggino, K.P.; Scollary, G.R. Wine bottle colour and oxidative spoilage: Whole bottle light exposure experiments under controlled and uncontrolled temperature conditions. Food Chem. 2013, 138, 2451-2459. [CrossRef] [PubMed]

13. Argyri, K.; Komaitis, M.; Kapsokefalou, M. Iron decreases the antioxidant capacity of red wine under conditions of in vitro digestion. Food Chem. 2006, 96, 281-289. [CrossRef]

14. Organisation Internationale de la Vigne et du Vin (OIV). Vine and Wine Outlook 2012; OIV: Paris, France, 2012.

15. Singleton, V.; Rossi, J.A. Colorimetry of total phenolics with phosphomolybdic phosphotungstic acid reagents. Am. J. Enol. Vitic. 1965, 27, 144-158.

16. Lee, J.; Durst, R.W.; Wrolstad, R.E. Determination of total monomeric anthocyanin pigment content of fruit juices, beverages, natural colorants, and wines by the $\mathrm{pH}$ differential method: Collaborative study. J. AOAC Int. 2005, 88, 1269-1278. [PubMed] 
17. Kim, Y.K.; Guo, Q.; Packer, L. Free radical scavenging activity of red ginseng aqueous extracts. Toxicology 2002, 172, 149-156. [CrossRef]

18. Re, R.; Pellegrini, N.; Proteggente, A.; Pannala, A.; Yang, M.; Rice-Evans, C. Antioxidant activity applying an improved ABTS radical cation decolorization assay. Free Radic. Biol. Med. 1999, 26, 1231-1237. [CrossRef]

19. Silva, J.K.; Cazarin, C.B.B.; Correa, L.C.; Batista, Â.G.; Furlan, C.P.B.; Biasoto, A.C.T.; Maróstica, M.R., Jr. Bioactive compounds of juices from two Brazilian grape cultivars. J. Sci. Food Agric. 2015, 96, 1990-1996. [CrossRef] [PubMed]

20. Hongyu, K.; Sandanielo, V.L.M.; Oliveira-Junior, G.J. Principal Component Analysis: Theory, interpretations and applications. Eng. Sci. 2015, 5, 1-8. [CrossRef]

21. Organisation Internationale de la Vigne et du Vin (OIV). Recueil des methods internationals d'analyse des vins et des mouts. In Proceedings of the 8th Assemblée Générale, Paris, France, 21 June 2010.

22. Sant'Anna, V.; Gurak, P.D.; Marczak, L.D.F.; Tessaro, I.C. Tracking bioactive compounds with colour changes in foods-A review. Dyes Pigment. 2013, 98, 601-608. [CrossRef]

23. Garrido, J.; Borges, F. Wine and grape polyphenols-A chemical perspective. Food Res. Int. 2013, 54, 1844-1858. [CrossRef]

24. Xing, R.R.; Li, L.Z.D.; Yuan, T.Y.; Zhang, X.X.; Li, J.M.; Pan, Q.H. Impact of different types of stoppers on sensorial and Phenolic characteristics evolution during a bottle storage time of a white wine from Chardonnay grape variety. J. Food Sci. Technol. 2016, 53, 4043-4055. [CrossRef] [PubMed]

25. Ribéreau-Gayon, P.; Glories, Y.; Maujean, A.; Dubourdieu, D. Tratado de Enologia 2: Quimica Del Vino, Estabilizacion y Tratamientos; Hemisferio Sur: Buenos Aires, Argentina, 2003.

26. Morata, A.; Loira, I.; Heras, J.M.; Callejo, M.J.; Tesfaye, W.; González, C.; Suárez-Lepe, J.Á. Yeast influence on the formation of stable pigments in red winemaking. Food Chem. 2016, 197, 686-691. [CrossRef] [PubMed]

27. Scrimgeour, N.; Nordestgaard, S.; Lloyd, N.D.R.; Wilkes, E.N. Exploring the effect of elevated storage temperature on wine composition. Aust. J. Grape Wine Res. 2015, 21, 713-722. [CrossRef]

28. Vincenzi, S.; Tomasi, D.; Gaiotti, F.; Lovat, L.; Giacosa, S.; Torchio, F.; Segade, S.R.; Rolle, L. Comparative Study of the Resveratrol Content of Twenty-one Italian Red Grape Varieties. S. Afr. J. Enol. Vitic. 2012, 34, 30-35. [CrossRef]

29. Guerrero, R.F.; Puertas, B.; Jiménez, M.J.; Cacho, J.; Cantos-Villar, E. Monitoring the process to obtain red wine enriched in resveratrol and piceatannol without quality loss. Food Chem. 2010, 122, 195-202. [CrossRef]

30. Gambuti, A.; Siani, T.; Picariello, L.; Rinaldi, A.; Lisanti, M.T.; Ugliano, M.; Dieval, J.B.; Moio, L. Oxygen exposure of tannins-rich red wines during bottle aging. Influence on phenolics and color, astringency markers and sensory attributes. Eur. Food Res. Technol. 2016, 243, 669-680. [CrossRef]

31. Kallithraka, S.; Salacha, M.I.; Tzourou, I. Changes in phenolic composition and antioxidant activity of white wine during bottle storage: Accelerated browning test versus bottle storage. Food Chem. 2009, 113, 500-505. [CrossRef]

32. Muselík, J.; García-Alonso, M.; Martín-López, M.P.; Žemlička, M.; Rivas-Gonzalo, J.C. Measurement of antioxidant activity of wine catechins, procyanidins, anthocyanins and pyranoanthocyanins. Int. J. Mol. Sci. 2007, 8, 797-809. [CrossRef]

33. Padilha, C.V.S.; Biasoto, A.C.T.; Corrêa, L.C.; Lima, M.S.; Pereira, G.E. Phenolic compounds profile and antioxidant activity of commercial tropical red wines (Vitis vinifera L.) from São Francisco Valley, Brazil. J. Food Biochem. 2017, 41, e12346. [CrossRef]

34. Cetó, A.X.; Apetreib, C.; Valle, M.; Rodríguez-Méndez, M.L. Evaluation of red wines antioxidant capacity by meansof a voltammetric e-tongue with an optimized sensor array. Electrochim. Acta 2014, 120, 180-186. [CrossRef]

(C) 2018 by the authors. Licensee MDPI, Basel, Switzerland. This article is an open access article distributed under the terms and conditions of the Creative Commons Attribution (CC BY) license (http:/ / creativecommons.org/licenses/by/4.0/). 